\title{
Rational Chebyshev Approximations for the Exponential Integral $E_{1}(x)$
}

\author{
By W. J. Cody* and Henry C. Thacher, Jr.**
}

Abstract. Rational Chebyshev approximations are presented for the exponential integral $E_{1}(x)$ in the intervals $(0,1],[1,4]$, and [4, $\left.\infty\right)$ with maximal relative errors ranging down to $10^{-21}$. $25 \mathrm{~S}$ coefficients are also given for a continued-fraction expansion for small $x$.

1. Introduction. Accurate function values for the exponential integral $E_{1}(x)$, defined [1] by

$$
E_{1}(x)=-\operatorname{Ei}(-x)=\int_{x}^{\infty} \frac{e^{-t}}{t} d t,
$$

are frequently needed in a variety of physical applications. Approximations to this function previously given by Allen [2] and Hastings [3], [4] are of insufficient accuracy for many such applications and for the longer word-length of modern computers. While Clenshaw [5] has given 20D coefficients for expansions in Chebyshev polynomials, such expansions are generally less efficient than rational forms. In addition, the Clenshaw form loses significance through subtraction for $1<x \leqq 4$. In this paper we present a set of nearly-best rational approximations for $E_{1}(x)$ for $0<x<\infty$ with accuracies up to 20S. The approximation forms used are efficient and are computationally stable.

2. Approximation Forms. The approximation forms and corresponding intervals used are

$$
\begin{aligned}
E_{l m}(x) & =-\ln (x)+R_{l m}(x), \quad 0<x \leqq 1, \\
& =e^{-x} R_{l m}(1 / x), \quad 1 \leqq x \leqq 4, \\
& =\frac{e^{-x}}{x}\left[1+\frac{1}{x} R_{l m}(1 / x)\right], \quad 4 \leqq x<\infty,
\end{aligned}
$$

where the $R_{l m}(z)$ are rational functions of degree $l$ in the numerator and $m$ in the denominator. All of the previously mentioned approximations have made use of the first and last of these forms, but not necessarily over the same intervals we use. The first form, based on the analytic expansion

$$
E_{1}(x)+\ln (x)=-\gamma-\sum_{n=1}^{\infty} \frac{(-x)^{n}}{n \cdot n !},
$$

where $\gamma$ is Euler's constant, involves a loss of significance through subtraction of

Received November 8, 1967.

* Work supported by the U. S. Atomic Energy Commission.

** Work supported in part by the U. S. Atomic Energy Commission and in part by the University of Notre Dame. 
nearly equal quantities when used for $x>1$. Thus, for example, Clenshaw loses up to three significant figures by using this form for $|x| \leqq 4$. The third form, based on the continued-fraction expansion of $E_{1}(x)$ for large $x$, is not efficient when used for the entire interval $[1, \infty)$. The combination of the second form and interval given above is the best of many such combinations tried.

3. Computations. Final computations were carried out in $25 \mathrm{~S}$ arithmetic on a CDC 3600. The approximations were computed without difficulty using standard versions of the Remes algorithm for rational Chebyshev approximation [6], [7]. Function values were computed as needed using one of two techniques, depending on the value of the argument. The expansion (3) converges for all $x$, but for $x$ appreciably greater than 1 , the rate of convergence is slow, and individual terms become large, causing subtraction errors. This series was transformed by means of

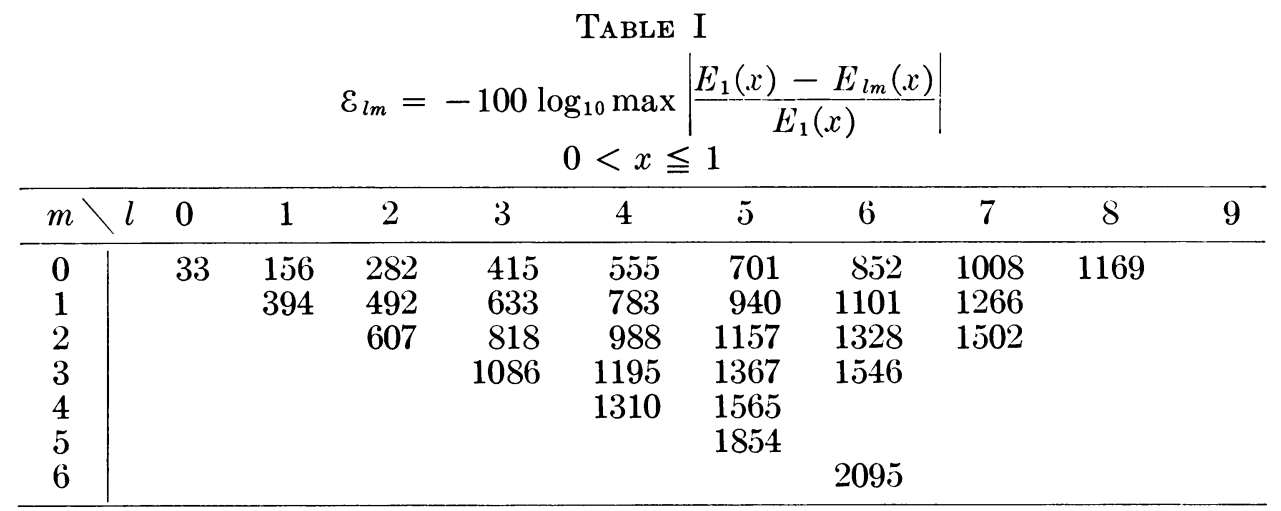

\begin{tabular}{|c|c|c|c|c|c|c|c|c|c|c|}
\hline \multicolumn{11}{|c|}{$1 \leqq x \leqq 4$} \\
\hline $\begin{array}{l}0 \\
1 \\
2 \\
3 \\
4 \\
5 \\
6 \\
7 \\
8\end{array}$ & 31 & $\begin{array}{l}145 \\
286 \\
386 \\
473 \\
554 \\
631\end{array}$ & $\begin{array}{l}242 \\
401 \\
531 \\
637 \\
732\end{array}$ & $\begin{array}{l}330 \\
503 \\
645 \\
769 \\
877\end{array}$ & $\begin{array}{r}412 \\
597 \\
749 \\
882 \\
1004\end{array}$ & $\begin{array}{r}491 \\
685 \\
846 \\
987 \\
1116 \\
1235\end{array}$ & $\begin{array}{r}567 \\
769 \\
937 \\
1086\end{array}$ & 641 & 713 & 783 \\
\hline \multicolumn{11}{|c|}{$4 \leqq x$} \\
\hline $\begin{array}{l}0 \\
1 \\
2 \\
3 \\
4 \\
5 \\
6 \\
7 \\
8 \\
9\end{array}$ & 193 & $\begin{array}{l}293 \\
458 \\
591 \\
687 \\
772\end{array}$ & $\begin{array}{l}382 \\
552 \\
691 \\
812 \\
906\end{array}$ & $\begin{array}{r}464 \\
639 \\
782 \\
909 \\
1022\end{array}$ & $\begin{array}{r}541 \\
720 \\
868 \\
998 \\
1116\end{array}$ & $\begin{array}{l}614 \\
797 \\
950\end{array}$ & 1512 & 1702 & 1888 & 2071 \\
\hline
\end{tabular}




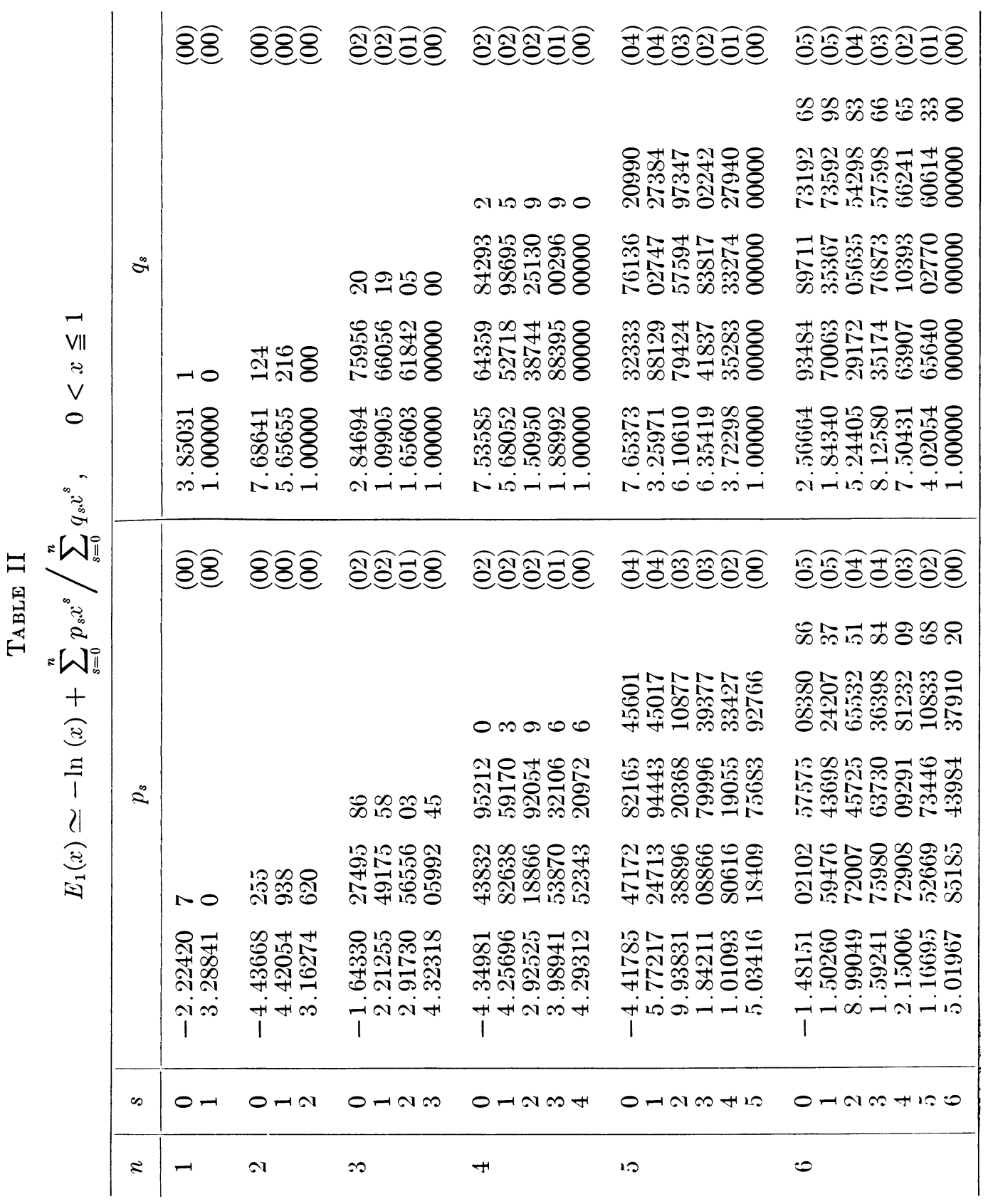




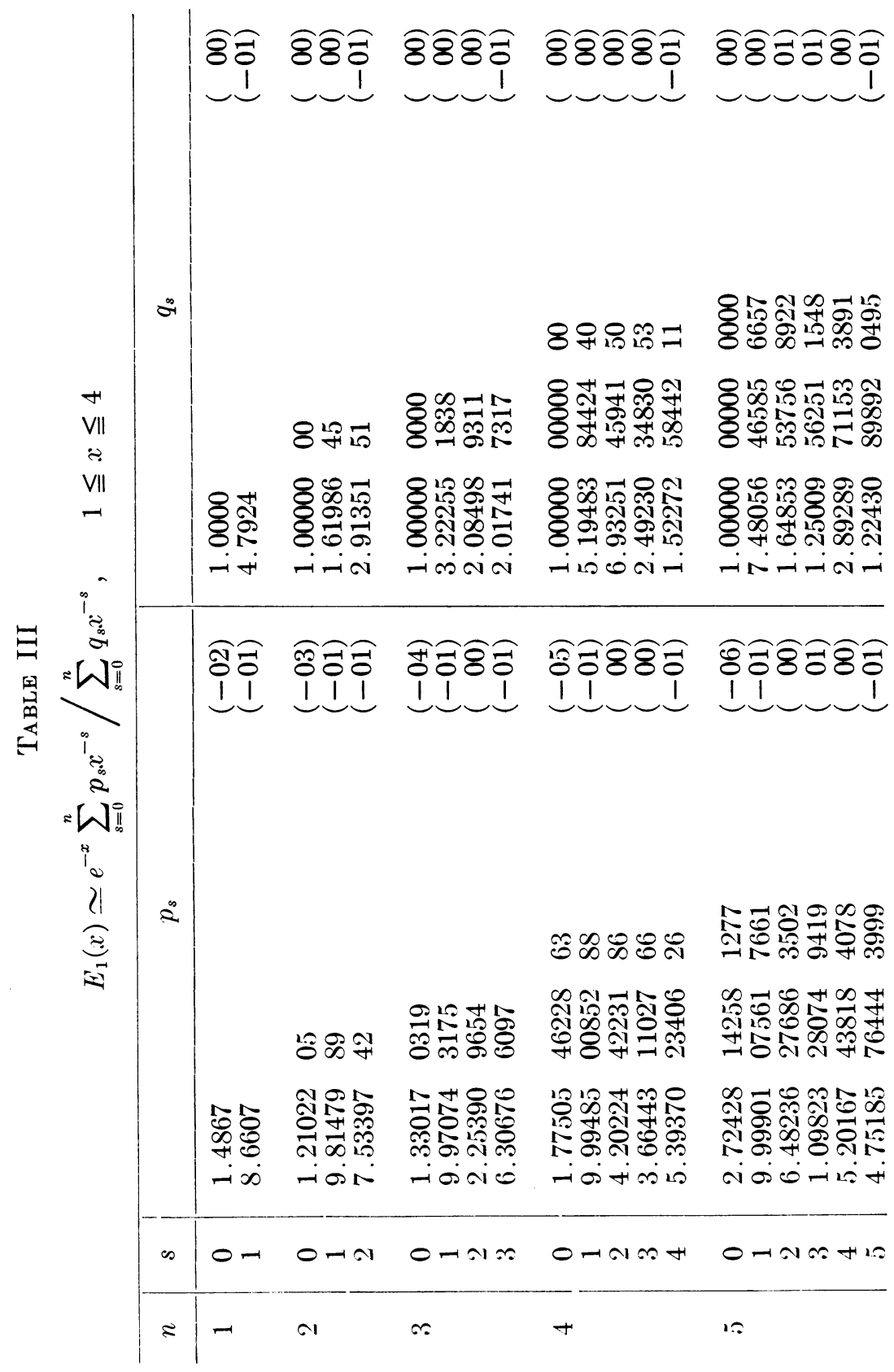




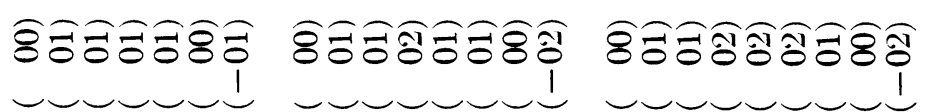
$0 m+m m n$ nom on

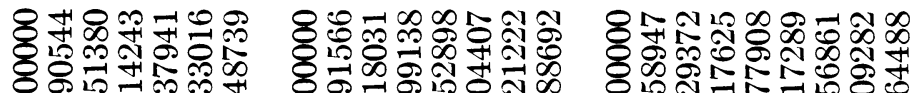

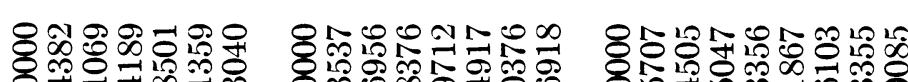

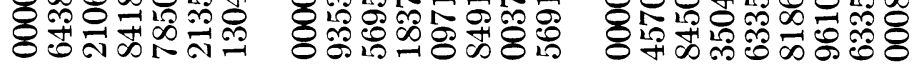

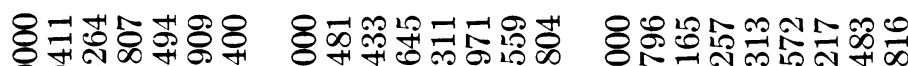

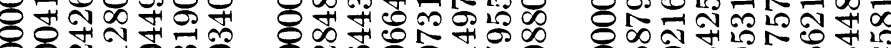

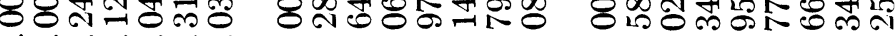

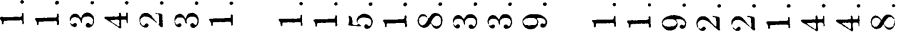

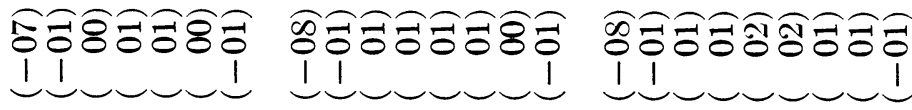
NNmeromeros \%

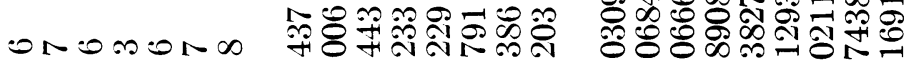

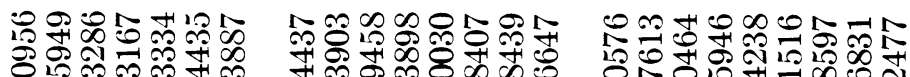

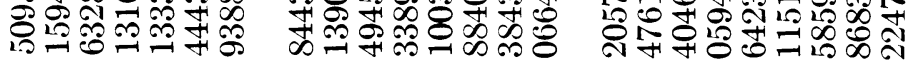

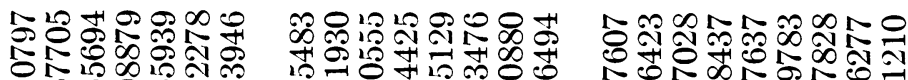

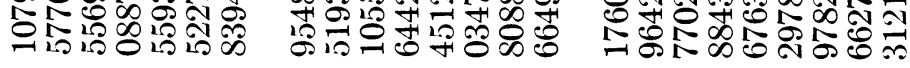

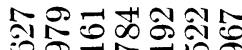

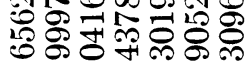

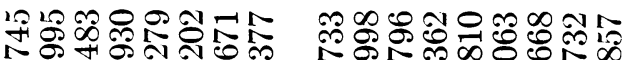

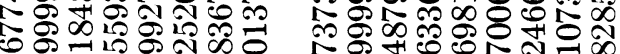
संबंबतं $\infty \dot{0}-\dot{+} \dot{0} \dot{0} \dot{+} \dot{0}$ -ion-

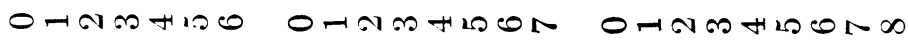
0 $N$ $\infty$ 


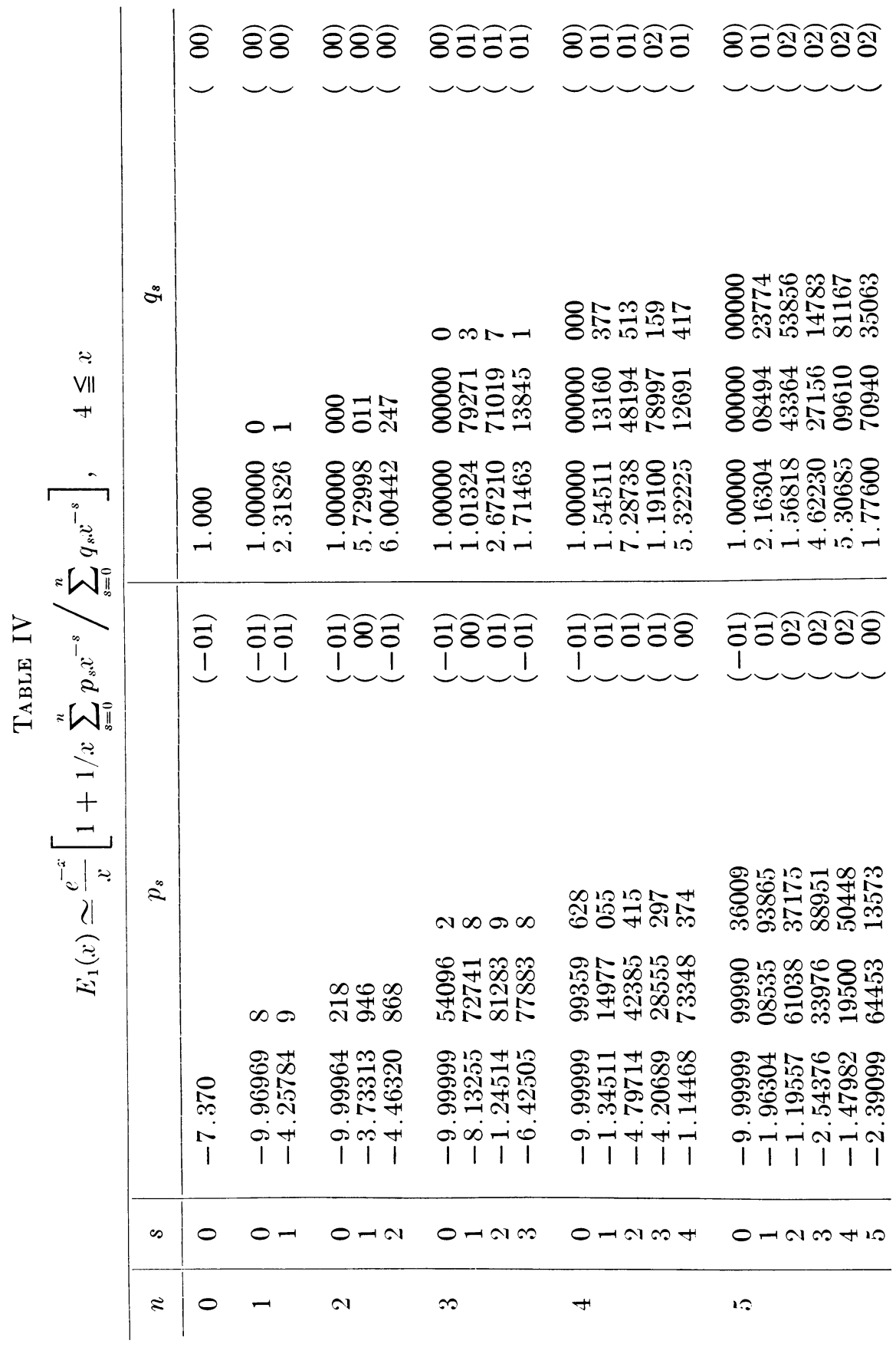




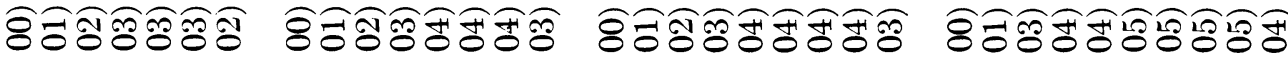

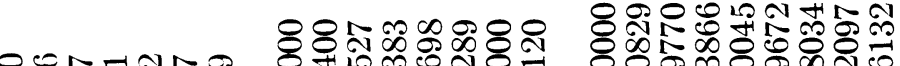

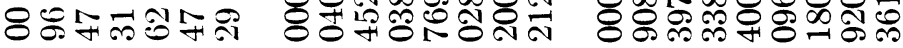

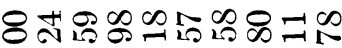

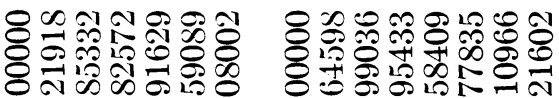

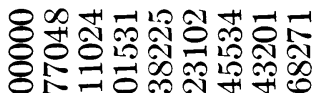

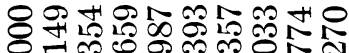

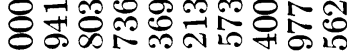

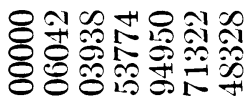

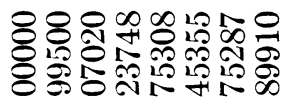

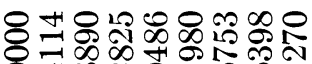

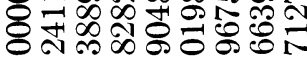

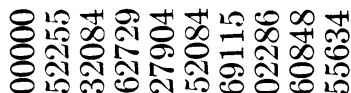

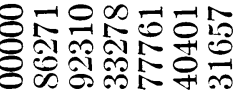

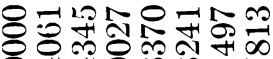

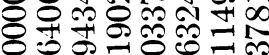
-NOTNOD

-

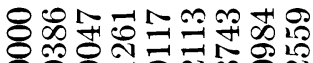

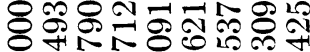
-íñó

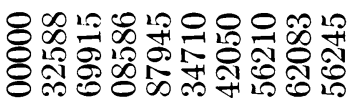

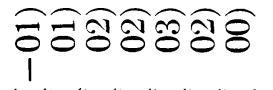

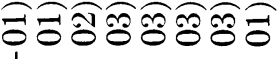

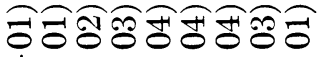
1

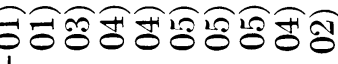
1

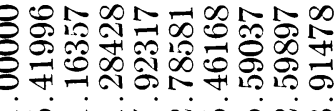

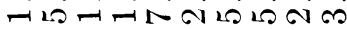

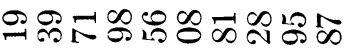

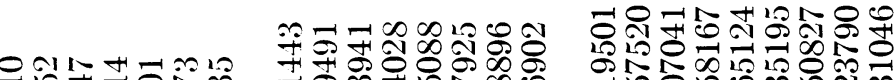

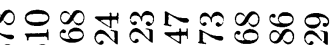

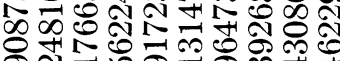

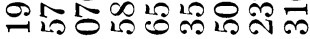

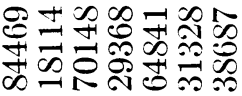

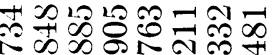

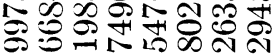

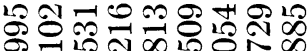

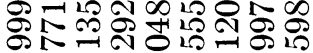

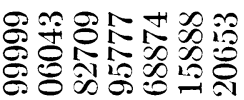

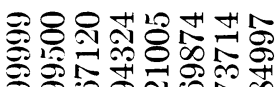

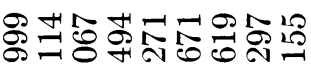

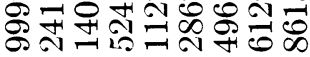

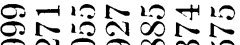

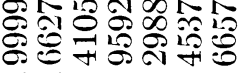

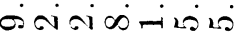

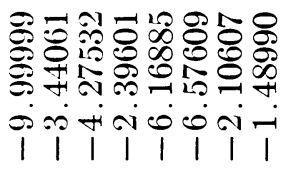

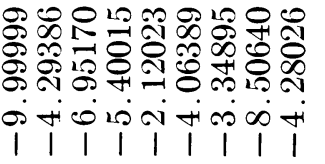

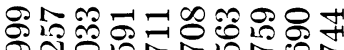

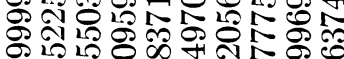
ร)

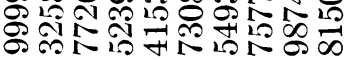

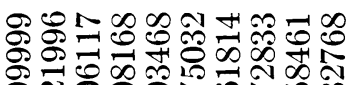
ริ के $\begin{array}{lllllll}1 & 1 & 1 & 1 & 1 & 1 & 1\end{array}$

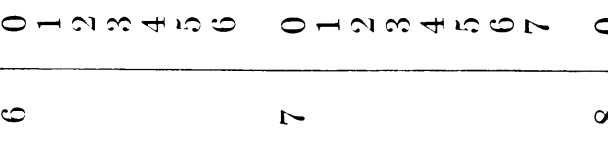

-

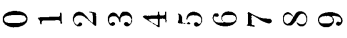


TABLE V

$$
E_{1}(x)=-\ln (x)-\gamma+\frac{a_{1} x}{1-} \frac{a_{2} x}{1-} \cdots
$$

\begin{tabular}{|c|c|c|c|c|c|c|}
\hline \multirow{2}{*}{$\frac{i}{1}$} & \multicolumn{6}{|c|}{$a_{i}$} \\
\hline & 1.00000 & 00000 & 00000 & 00000 & 0000 & $(00)$ \\
\hline 2 & -2.50000 & 00000 & 00000 & 00000 & 0000 & $(-01)$ \\
\hline 3 & 2.77777 & 77777 & 77777 & 77777 & 7778 & $(-02)$ \\
\hline 4 & -2.77777 & 77777 & 77777 & 77777 & 7778 & $(-01)$ \\
\hline 5 & 1.64000 & 00000 & 00000 & 00000 & 0000 & $(-01)$ \\
\hline 6 & -4.81463 & 41463 & 41463 & 41463 & 4146 & $(-02)$ \\
\hline 7 & 1.86603 & 29429 & 42170 & 56679 & 5923 & $(-02)$ \\
\hline 8 & -1.60205 & 68757 & 07501 & 54525 & 5703 & $(-01)$ \\
\hline 9 & 1.23861 & 39210 & 41923 & 32991 & 7815 & $(-01)$ \\
\hline 10 & -2.31794 & 05112 & 45808 & 29915 & 6950 & $(-02)$ \\
\hline 11 & 1.29681 & 11650 & 66612 & 32574 & 9017 & $-(02)$ \\
\hline 12 & -1.12427 & 07106 & 81348 & 55498 & 0946 & $(-01)$ \\
\hline 13 & 9.48210 & 68248 & 17627 & 18042 & 2609 & $(-02)$ \\
\hline 14 & -1.50705 & 56940 & 70993 & 41586 & 7282 & $(-02)$ \\
\hline 15 & 9.91703 & 79965 & 35417 & 38979 & 0346 & $(-03)$ \\
\hline 16 & -8.65217 & 39735 & 62029 & 78930 & 2325 & $(-02)$ \\
\hline 17 & 7.61891 & 65113 & 71489 & 38461 & 1831 & $(-02)$ \\
\hline 18 & -1.11260 & 95393 & 81208 & 17324 & 8394 & $(-02)$ \\
\hline 19 & 8.02137 & 38225 & 66099 & 94103 & 6526 & $(-03)$ \\
\hline 20 & -7.03079 & 19046 & 68019 & 59276 & 8798 & $(-02)$ \\
\hline 21 & 6.35254 & 92288 & 39041 & 18840 & 0415 & $(-02)$ \\
\hline 22 & -8.80574 & 51173 & 87599 & 83602 & 9885 & $(-03)$ \\
\hline 23 & 6.73156 & 43159 & 35130 & 57355 & $341 \tilde{1}$ & $(-03)$ \\
\hline 24 & -5.92093 & 68551 & 88450 & 02360 & 8312 & $(-02)$ \\
\hline 25 & 5.44193 & 72539 & 14569 & 37403 & 6161 & $(-02)$ \\
\hline 26 & -7.28137 & 18497 & 92553 & 59228 & 5206 & $(-03)$ \\
\hline 27 & 5.79799 & 42107 & 94125 & 58486 & 8766 & $(-03)$ \\
\hline 28 & -5.11362 & 97085 & 60959 & 55012 & 6918 & $(-02)$ \\
\hline 29 & 4.75746 & 03552 & 29856 & 08437 & 2345 & $(-02)$ \\
\hline 30 & -6.20470 & 20824 & 22709 & 71921 & 3328 & $(-0.3)$ \\
\hline 31 & 5.09130 & 38779 & 10156 & 55165 & 2889 & $(-03)$ \\
\hline 32 & -4.50002 & 85546 & 37539 & 73594 & $709 S$ & $(-02)$ \\
\hline 33 & 4.22487 & 22218 & 42761 & 47612 & 1945 & $(-02)$ \\
\hline 34 & -5.40431 & 89612 & 94834 & 49318 & 0440 & $(-03)$ \\
\hline 35 & 4.53788 & 95713 & 23179 & 68530 & 3408 & $(-03)$ \\
\hline 36 & -4.01789 & 51290 & 74937 & 23947 & 5927 & $(-02)$ \\
\hline 37 & 3.79896 & 24606 & 33396 & 75543 & 1572 & $(-02)$ \\
\hline 38 & -4.78623 & 11965 & 44072 & 42723 & $697 \bar{j}$ & $(-03)$ \\
\hline 39 & 4.09283 & 20621 & 35569 & 26450 & $881 j$ & $(-03)$ \\
\hline 40 & -3.62907 & 03638 & 13851 & 03882 & 9943 & $(-02)$ \\
\hline
\end{tabular}


the QD algorithm [8], using 40S arithmetic, into a continued fraction

$$
E_{1}(x)+\ln (x)=-\gamma+\frac{a_{1} x}{1-} \frac{a_{2} x}{1-} \frac{a_{3} x}{1-} \cdots
$$

which converges rapidly with little inherent error except near the zero at $x \simeq .675$. Using this continued fraction for $0<x \leqq 4$, and the standard continued fraction [1, Eq. 5.1-22] for $x>4$, at least 23S master function values were obtained for $E_{1}$ for all $x>0$.

4. Results. Table I lists the values of

$$
\mathcal{E}_{l m}=-100 \log _{10} \max \left|\frac{E_{1}(x)-E_{l m}(x)}{E_{1}(x)}\right|,
$$

where the maximum is taken over the appropriate interval, for the initial segments of the various $L_{\infty}$ Walsh arrays. Tables II-IV present the coefficients for the main diagonals of these arrays. All coefficients are given to accuracies greater than that justified by the maximal errors, but reasonable additional rounding should not greatly affect the overall accuracies. Each approximation listed, with the coefficients rounded as they appear in the table, was tested against the master function routines for 5000 pseudo-random arguments. In all cases maximal errors agreed, within roundoff, in magnitude and location with those for the unrounded approximations.

Table $\mathrm{V}$ lists the coefficients of the continued fraction (4) to $25 \mathrm{~S}$. While these coefficients are not all correct to $25 \mathrm{~S}$, the evaluation of (4) using these coefficients appears correct to nearly $25 \mathrm{~S}$ for $x \leqq 8$, (25D for $x \simeq .675)$, slowly deteriorating in accuracy for larger $x$.

Applied Mathematics Division Argonne National Laboratory Argonne, Illinois 60439

Computing Science Department

University of Notre Dame

Notre Dame, Indiana 46556

1. W. GAtrschi \& W. F. CAhill, "Exponential integral and related functions," Chapter j in Handbook of Mathematical Functions with Formulas, Graphs and Mathematical Tables, edited by M. Abramowitz \& I. A. Stegun, Nat. Bur. Standards Appl. Math. Series, 55, Superintendent of Documents, U. S. (iovernment Printing Office, Washington, D. C., 1964; 3rd printing, with corrections, 1965. MP 29 \#4914; MR $31 \# 1400$.

2. E. E. Allen, "Note 169," MTAC, v. 8, 1954, p. 240.

3. C. HAstings, JR., A pproximations for Digital Computers, Princeton Univ. Press, Princeton, N. J., 1955, pp. 188-190. \IR 16, 963.

4. C. Hastings, JR., "Note 143 ," $M T A C$, v. 7, 1953, p. 68.

5. C. W. Clenshaw, Chebyshev Series for Mathematical Functions, National Physical Laboratory Math. Tables, Vol. 5, Department of Scientific and Industrial Research, H.M.S.O., London, 1962. MR $26 \# 362$.

6. W. FrASER d J. F. HART, "On the computation of rational approximations to continuous functions," Comm. ACM, v. 5, 1962, pp. 401-403.

7. W. J. Cody \& J. SToer, "Rational Chebyshev approximations using interpolation," Numer. Math., v. 9, 1966, pp. 177-188.

8. P. HeNrici, "Some applications of the quotient difference algorithm" in High Speed Computing and Experimental Arithmetic, Proc. Sympos. Appl. Math., Vol. 15, Amer. Math. Soc., Providence, R. I., 1963, pp. 159-183. MR 28 \#2632. 